\title{
El desarrollo de competencias digitales en la educación superior
}

\author{
Mario Arias Oliva \\ Universitat Rovira I Virgili \\ mario.arias@urv.cat \\ Teresa TORRES CORONAS \\ Universitat Rovira I Virgili \\ teresa.torres@urv.cat \\ Juan Carlos YÁÑEZ LuNA \\ Universidad Autónoma de San Luis Potosí \\ jcyl@usalp.mx
}

\begin{abstract}
Resumen:
El presente estudio pretende determinar si las competencias adquiridas por parte de los alumnos de grado de la Universitat Rovira i Virgili convergen con los factores que pudieran definir los nuevos "nativos digitales". A través de una revisión bibliográfica concluimos que los alumnos cuentan con las habilidades necesarias. Creemos que la universidad debe de tomar parte en el desarrollo de estas competencias, incluyéndolas un entorno holístico en el proceso de aprendizaje.
\end{abstract}

Palabras clave: Competencias digitales; Web 3.0; e-Learning

\section{Development of digital skills in higher education}

\begin{abstract}
:
Our research focus on skills acquired by degree students in Universitat Rovira i Virgili. We analyze competences in ICT area to determine if students are "digital natives". Our literature review concluded ICT use is a critical competence nowadays. We conclude that universities should take part in the development of these skills by developing a holistic learning environment based on technological tools.
\end{abstract}

Key Words: Digital skills; Web 3.0; e-Learning

Referencia normalizada:

Arias Oliva, M., Torres Coronas, T. y Yañes Luna, J.C. (2014): El desarrollo de competencias digitales en la educación superior. Historia y Comunicación Social. Vol. 19. Núm. Especial Enero. Págs. 355-366.

Sumario: 1. Introducción, 1.1. Evolución de la formación: una mirada hacia el e-Learning, 1.2. La web: colaboración y semántica, 1.3. Proceso educativo y las TIC, 2. Habilidades y capacidades: competencias, 3. Competencias digitales en la educación superior: caso Universitat Rovira I Virgili, 3.1. Las competencias digitales en la URV, 4 . Conclusiones. 


\section{Introducción}

Actualmente la incorporación de las Tecnologías de Información y Comunicaciones (TIC) dentro de la sociedad ha cambiado completamente la percepción del aprendizaje y de la comunicación. Podemos pensar que los cambios económicos provocados por la globalización y los cambios emergentes en la industria han requerido que las universidades formen nuevos profesionales con competencias basadas en el uso de las TIC. Con la evolución continua entre las tecnologías (hardware y software) los usuarios están divididos en dos grupos: Los que nacen con las TIC y los que requieren entrenamiento para su uso.

En este sentido, Chisega-Negrila (2012:459) apunta a que los cambios se enfocan a una adquisición de información más efectiva por parte de las instituciones académicas, logrando con ello una mejor construcción el conocimiento. Sin embargo el problema puede radicar en el saber utilizar la herramienta para disgregar la información confiable de la que no lo es, tal como lo argumenta Loureiro (2012:537). Por lo tanto también el alumno debe adquirir las capacidades necesarias que requieren sus actividades de aprendizaje y posteriormente el mercado laboral como recogen Martínez Alvarado (2009:62); Camacho \& Lara (2011:153). Ampliando el concepto anterior, Santiago Campión \& Navaridas Nalda (2012:28) apuntan a que los profesores deben de tener ciertas cualidades para la utilización de la Web 3.0 de tal manera que puedan encaminar al alumnado hacia la construcción del conocimiento.

El presente estudio pretende determinar si las competencias adquiridas por parte de los alumnos de grado de la Universitat Rovira I Virgili convergen con los factores que pudieran definir los nuevos "nativos digitales".

\subsection{Evolución de la formación: una mirada hacia el E-learning}

La educación a distancia se ha enfrentado a diversos cambios a través del tiempo, provocados en numerosas ocasiones por los avances tecnológicos. Actualmente las telecomunicaciones están en un proceso de rediseño e innovación continuo, asistiendo a un una continua e innovación y tanto en dispositivos como en servicios de comunicación.

El proceso educativo no queda fuera de esta concepción de cambios. Se observa que:

Es importante que la IoT1 se convierta en parte de la conversación estratégica actual y futura, ya sea a nivel de las instituciones de investigación o discusiones de cooperación internacional para asegurar que cosechamos los beneficios presentados por la IoT. (Coetzee \& Eksteen 2011:9)

Por lo tanto el trabajar con las TIC (revolucionando el espacio-tiempo-medio dentro o fuera del aula) en los espacios académicos, puede ser positivamente significante hacia la concepción de competencias digitales, habilidades tecnológicas y a su vez el tipo de comunicación que se da entre alumnos y alumno-docente. Consideramos 
entonces que los ambientes virtuales formarán el enlace o la red social intra-escolar que fortalecerán esta comunicación y que favorecerán al aprendizaje.

En general la evolución se ha dado con base a los modelos ya existentes. Sin embargo, en la reutilización de estos modelos se abren nuevos conceptos híbridos para este tipo de formación a distancia: Blended Learning (b-Learning), acciones formativas mixtas, combinan la formación presencial y la formación en línea intentando combinar los mejores aspectos de cada modelo, y el Mobile Learning (mLearning), aplicación de las tecnologías móviles al eLearning, una metodología de enseñanza y aprendizaje a través del uso de dispositivos móviles (Fernández Gómez 2009:33-34).

\subsection{La web: colaboración y semántica}

Dentro del entorno de colaboración por Internet, encontramos sin lugar a dudas una evolución importante dentro de Internet: es la Web 2.02. La Web 2.0 "La revolución comercial en la industria informática causada por la transformación de Internet como plataforma, y un intento de entender las normativas para el éxito en esa nueva plataforma" definida por (O'Reilly 2006:1). Otro concepto define a la Web 2.0 como "la transición que se ha dado de aplicaciones tradicionales hacia aplicaciones que funcionan a través de la Web enfocadas al usuario final" (Fernández Gómez 2009:45).

El argumento anterior determina el contexto tecnológico en Internet, de acuerdo con Prendes Espinoza (2007:215,216), el uso de estas plataformas potencializan el perfeccionamiento de contextos colaborativos incorporando Chat, Wiki's, WebQuest, Blogs, correos electrónicos, herramientas desarrolladas para uso exclusivo de este tipo de enseñanza en red tales como los campus virtuales, LMS, LCMS y EVEAs, Groupware, CSCL, CSCW3 y Mundos Virtuales 3D.

Sin embargo, la evolución de la Web también ha sido en torno a las TIC. El concepto de Web 3.0 es visto como "una extensión de la WWW y de la Web 2.0, en la que la información puede ser compartida e interpretada por otro agente de software para encontrar e integrar aplicaciones en diferentes dominios" (Padma \& Seshasaayee 2011:420).

La evolución marcará entonces una nueva manera de acceder a la información. En este sentido no solo se basará en búsquedas de información en sitios Web, sino más bien en base a los Tags o etiquetas. Chisega-Negrila (2012:456) señala que "el elemento de novedad traída por la Web Semántica es la adaptación del contenido a un usuario específico", es decir, adaptado para el usuario que requiere la información.

Dentro de este plano evolutivo, consideramos que es muy importante profesionalizar los contenidos de los sitios Web y de los Recursos de Educación Abiertos. En este sentido Lehmann y otros (2013) identifican en su estudio a usuarios denominados "News Curators" quienes se encargan de validar la información sobre temas específicos.

Por otro lado en el estudio de Morris (2011:44) se observa que pueden existir una serie de implicaciones en la adopción de la Web 3.0 dentro de un contexto de educa- 
ción en línea. Por consiguiente creemos que estas implicaciones podrían deberse también a la falta de adquisiciones de competencias tecnológicas que les permitan tanto a docente como alumnos adaptarse a un entorno de acceso de información distinto y en cambio constante.

\subsection{Proceso educativo y las TIC}

La transformación de los sistemas educativos a distancia se encamina hacia una estrategia comercial y cultural. El mercado de las TIC se ha convertido claramente en una economía de escalas. En este sentido, con la multivariada gama de dispositivos de telecomunicaciones fabricados y el fácil acceso que se tienen a ellos se hacen posible la transformación del Internet de ordenadores al Internet de cosas. En este concepto, se visualiza una nueva manera de enfrentar los enfoques de comunicación, es decir; cada uno de los dispositivos e Internet se fusionan en uno solo.

Por lo tanto, la innovación en los enfoques educativos haciendo uso de las TIC, permiten al usuario4 estar en contacto con diversas aplicaciones y otros dispositivos sin importar el tiempo, distancia o medios en entornos de comunicación síncrona o asíncrona. Por ello el uso de estas tecnologías está fomentando el desarrollo de nuevos entornos de investigación, como lo menciona González Mariño (2008:3) "Las innovaciones en educación tienen ante sí como principal reto los procesos de adopción por parte de las personas, los grupos y las instituciones". Estos retos se centran en el entorno del prefijo "E-" y los conceptos emergentes del mismo.

En el contexto de centros educativos, estudios como los realizados por Cacciamani y otros (2012); Guzmán Flores y otros (2011); González (2010); Benson y Brack (2009); Stevens (2008) plantean mejorar el desarrollo de contenidos, al papel de la TICs en la mejora de los procesos de aprendizaje, enseñanza y generación del conocimiento, refiriéndose también al entrenamiento y formación de profesores y tutores de programas virtuales englobados en un concepto denominado "e-Teaching". En este sentido, (Jethro et al. 2012; Domingo \& Marquès 2011; Guzmán Flores et al. 2011; Reyes González \& Guevara Cruz 2009; Hermes Lück 2009; Cabero et al. 2003), observan que la integración del eLearning dentro del sistema educativo transforma también la actividad de los educadores, es decir, el profesor o tutor dejará de ser un distribuidor de contenido y fungirá como un facilitador de aprendizaje y asesor de competencias.

En este concepto (Roldán y otros 2010:19) definen al profesor-tutor como: "el encargado de llevar el peso académico, debe ser experto en la materia a impartir y conocer las posibilidades y limitaciones del sistema para poder aprovechar o reforzar los contenidos según corresponda", mientras que el administrador del sistema debe de atender las necesidades de los profesores mediante el funcionamiento del entorno electrónico de formación.

En el marco de la formación docente Hermes Lück (2009:9) concluye que se vincula una perspectiva crítica sobre el conocimiento del docente, sus procesos y sus estrategias pedagógicas y sobre el desafío de mejorar su práctica educativa con 
coherencia y en sintonía con las exigencias y necesidades de la sociedad o el entorno en el que se desenvuelve el individuo.

Otras temáticas dentro del entorno educativo han llevado a diversos investigadores a estudiar el comportamiento, sentido de identidad cultural y conducta del estudiante dentro de un entorno de enseñanza (Marques y Belo, 2011; Penalva Martínez, Rey Más, y Llinares Ciscar , 2011; Lee, Hsieh, y Hsu, 2011; De la Fuente Arias y otros, 2010; Yeh, 2010). Estos estudios se enfocan en determinar el grado de satisfacción o aceptación de las nuevas metodologías de enseñanza en clase por parte de los estudiantes. Por lo tanto, el objetivo de la educación online es interesar a la sociedad hacia la integración de las TIC en el proceso de aprendizaje; como una nueva forma de enseñanza a través de entornos virtuales.

Por otro lado, dentro del entorno empresarial se comenzó a instruir este tipo de didácticas bajo el apelativo de e-Training y su principal objetivo era el fortalecimiento de las cualidades y habilidades de sus empleados con el fin de llevar a cabo nuevas tareas o competencias (Byun y Mills, 2011; Hunt y otros, 2011; Penalva Martínez, Rey Más, y Llinares Ciscar, 2011). En este sentido se puede observar que la relación entre las actividades académicas y la incorporación de las TIC en el proceso de enseñanza se relacionan con las necesidades de fortalecer las habilidades y cualidades de los alumnos para su mejor integración en el ámbito laboral.

\section{Habilidades y capacidades: competencias}

Internet ha sido la herramienta que indudablemente ha revolucionado la manera de realizar la mayoría las actividades de los individuos. La manera en que hemos sido alfabetizados ha cambiado a lo largo del tiempo sin lugar a dudas.

En este sentido, cabe señalar que actualmente no solo basta con saber buscar y acceder a la información sino también saber desenvolverse en el ámbito tecnológico, tal como lo atribuye Area \& Guarro (2012:68)

En este nuevo medioambiente sobreviven y crecen aquellos individuos o colectivos sociales que dispongan de las competencias para producir, difundir y consumir información de forma rápida, eficaz y eficiente, es decir, de forma exitosa para desenvolverse como sujeto socializado. Y para ello, es fundamental e imprescindible saber transformar la información en conocimiento, disponer de las habilidades y capacidades para utilizar de forma eficiente los recursos y herramientas tanto de búsqueda de información como de producción y difusión de la misma, así como para comunicarla y compartirla socialmente a través de las distintas herramientas y entornos digitales.

Entendemos entonces por habilidades y capacidades de un individuo como una referencia a las competencias. Una competencia se define como "la capacidad para el desempeño con éxito de funciones y papeles en un ámbito específico de la actividad humana o en el marco general de la vida" (De la Orden Hoz 2011:47). 
Sin embargo existen entre las instituciones ciertas ambigüedades con respecto a la efectividad de la adquisición de competencias en los cursos online. En este sentido López de la Madrid \& Flores Guerrero (2006:50) afirman que los cursos en línea son tan valiosos como los cursos presenciales. Esto es, que las competencias que se requieren dentro de la formación presencial y online son las mismas competencias metodológicas y tecnológicas que requieren en un contexto laboral.

\section{Competencias digitales en la educación superior: caso Univertitat Rovira I Virgill}

Cartelli (2010:573) concluye en su trabajo que las clasificaciones utilizadas hasta ahora han sido las clásicas: "el desarrollo cognitivo, el afectivo y el psicomotor". También refiere a que con la llegada de las TIC se ha introducido al proceso de aprendizaje nuevos elementos como "las relaciones sociales, asociación y colaboración por medio de comunidades". Por lo tanto se expone que estos nuevos elementos serán una contribución esencial para el desarrollo de habilidades en el uso de estas

Las competencias digitales están fuertemente ligadas a los servicios que proporcionen dentro de las actividades OnLine. En este sentido Arias-Oliva \& Yáñez-Luna (2013:29) definen el eLearning como:

El proceso de creación, preparación, funcionamiento y distribución del conocimiento a través de metodologías de enseñanza y contenido distribuido a través del uso de las TIC en colaboración y / o de forma individual para superar las limitaciones estructurales de tiempo y espacio. Creemos que el proceso de aprendizaje se debe considerar de manera integral, donde las TIC es sólo una frontera entre el tradicional Learning "cara a cara" de aprendizaje y los modelos emergentes de eLearning online. La frontera de distinguir entre el aprendizaje con todas las herramientas TIC (tabletas, LMS, etc) y los métodos tecnológicos no tradicionales (pizarra, clase magistral, libros, lecturas, etc).

De esta manera, para que los estudiantes online puedan adquirir las competencias que el sector laboral demanda, las instituciones académicas deben de transformar el proceso de aprendizaje. En este sentido, (Torres- Coronas y otros 2013:471) visualizan que dentro de las iniciativas empresariales comienza a relacionarse esta evolución de las TIC con las transformaciones y cambios de paradigmas en la sociedad del conocimiento.

Por lo tanto, los sistemas de educación y formación deben generar esas nuevas competencias. A estas competencias debe de incluirse las competencias básicas y el aprendizaje como requisitos previos a la adquisición de mayores habilidades Ibid:472. Con ello logramos mejores oportunidades de incorporación y adaptación al campo laboral tanto de los nuevos como los que ya se encuentran dentro del sistema. Así pues, los estudiantes requerirán de una mayor solidez en sus habilidades y en el conocimiento adquirido. Las instituciones de educación superior deberían tratar de cubrir las habilidades y competencias TIC y, al mismo tiempo, deben desarrollar 
competencias digitales y tecnológicas entre sus alumnos. Cualquier institución de educación superior tiene que supervisar continuamente las perspectivas económicas y de empleo y ajustar los programas de la universidad correspondiente.

El presente estudio pretende determinar si las competencias adquiridas por parte de los alumnos de grado de la Universitat Rovira I Virgili convergen con los factores que pudieran definir a la Web 3.0, de igual manera determinar si estos factores de la Web 3.0 giran en torno a un modelo de eLearning. El marco competencial URV divide las competencias de los estudiantes en tres tipos: específicos, transversales y nucleares. Las competencias específicas están relacionadas con el conocimiento (saber y entender) y habilidades (saber actuar) de cada grado, no siendo transferibles a otros campos profesionales. Las competencias transferibles se refieren a actitudes y valores (saber ser), y procedimientos (saber hacer), que pueden ser transferidas de un campo profesional específico a otro. Por último, las competencias básicas son las competencias requeridas por los estudiantes URV, que pueden ser los conocimientos, actitudes y procedimientos, dentro de las cuales situamos las competencias digitales.

\subsection{Las competencias digitales en la URV}

El EEES es un modelo de educación con un enfoque basado en competencias, que tiene como objetivo garantizar que las competencias que se enseñan son las que se requieren en el lugar de trabajo. Las competencias que permiten estudiante tener éxito y tener un título universitario, también les permitirá prosperar en sus carreras profesionales. El marco competencial URV se implementó por primera vez en el curso 2003 / 04 para adaptar sus planes de estudio al EEES. El marco de competencias propuesta divide las competencias de los estudiantes en tres tipos:

- Específicos;

- Transversales o transferibles

- Nuclear o núcleo.

Dentro de cada competencia, se pueden formular los distintos niveles de dominio. Estos niveles de expresar el grado de consecución de una competencia a lo largo de un grado, un curso o una actividad planificada. Como una propuesta de trabajo inicial, la URV ha establecido las competencias básicas y transferibles de una variedad de disciplinas.

La Tabla 1 muestra los resultados de un estudio realizado por Torres- Coronas y otros (2013). Se evalúan las competencias digitales de estudiantes de la Universidad Rovira i Virgili con el propósito de conocer si se están desarrollando las mismas. 
Tabla 1. Competencia digital nivel entre estudiantes URV a. Diferencias estadísticas significativas, $\mathrm{p}<0.05$

\begin{tabular}{|c|c|c|c|c|c|c|c|c|c|c|}
\hline & \multicolumn{2}{|c|}{$\begin{array}{l}\text { Total Muestra } \\
(\mathrm{N}=578)\end{array}$} & \multicolumn{2}{|c|}{$\begin{array}{c}\text { Mujer } \\
(\mathbf{n}=304)\end{array}$} & \multicolumn{2}{|c|}{$\begin{array}{l}\text { Hombre } \\
(\mathrm{n}=274)\end{array}$} & \multicolumn{2}{|c|}{$\begin{array}{c}\text { Ciencias Sociales } \\
(\mathrm{n}=290)\end{array}$} & \multicolumn{2}{|c|}{$\begin{array}{c}\text { Ingeniería } \\
(\mathrm{n}=288)\end{array}$} \\
\hline & MEDIA & $\begin{array}{l}\text { DESV. } \\
\text { STD }\end{array}$ & MEDIA & $\begin{array}{l}\text { DESV. } \\
\text { STD }\end{array}$ & MEDIA & $\begin{array}{l}\text { DESV. } \\
\text { STD }\end{array}$ & MEDIA & $\begin{array}{l}\text { DESV. } \\
\text { STD }\end{array}$ & MEDIA & $\begin{array}{l}\text { DESV. } \\
\text { STD }\end{array}$ \\
\hline $\begin{array}{l}\text { l - Capacidad para utilizar las herramientas } \\
\text { electrónicas interactivas y no sólo de correo } \\
\text { electrónico asincrónica para comunicarse (por } \\
\text { ejemplo, Skype) }\end{array}$ & 4.01 & 0.99 & $4.11 \mathrm{a}$ & 0.95 & $3.89 \mathrm{a}$ & 1.03 & 4.04 & 0.99 & 3.97 & 0.99 \\
\hline $\begin{array}{l}2 \text { - Capacidad para utilizar las herramientas } \\
\text { electrónicas y de software específicos para } \\
\text { apoyar la colaboración en equipo virtual (por } \\
\text { ejemplo, Google docs) }\end{array}$ & 3.09 & 0.99 & 3.12 & 0.95 & 3.05 & 1.05 & 3.02 & 0.95 & 3.15 & 1.04 \\
\hline $\begin{array}{l}3 \text { - Capacidad para fomentar la e-colaboración y } \\
\text { el intercambio de ideas con los demás (por } \\
\text { ejemplo, e-liderazgo) }\end{array}$ & 2.2 & 1.23 & 2.26 & 1.22 & 2.14 & 1.23 & 2.19 & 1.18 & 2.22 & 1.27 \\
\hline $\begin{array}{l}4 \text { - Capacidad de estilo de la comunicación } \\
\text { electrónica a medida para adaptarse a la } \\
\text { audiencia y a la situación (el diseño y desarrollo } \\
\text { de blogs por ejemplo) }\end{array}$ & 3.5 & 1.27 & $3.61 \mathrm{a}$ & 1.24 & $3.37 \mathrm{a}$ & 1.29 & 3.52 & 1.25 & 3.48 & 1.3 \\
\hline $\begin{array}{l}5 \text { - Capacidad de organizar un equipo virtual y } \\
\text { asignar tareas y responsabilidades para lograr los } \\
\text { objetivos }\end{array}$ & 3.47 & 0.95 & $3.37 \mathrm{a}$ & 0.9 & $3.58 \mathrm{a}$ & 0.99 & 3.4 & 0.93 & 3.53 & 0.96 \\
\hline $\begin{array}{l}6 \text { - Capacidad de busca y evaluar e-fuentes de } \\
\text { información para resolver problemas y la toma } \\
\text { de decisiones }\end{array}$ & 3.97 & 0.84 & 4.03 & 0.82 & 3.9 & 0.86 & 3.93 & 0.79 & 4 & 0.89 \\
\hline $\begin{array}{l}7 \text { - Capacidad de gestión de la seguridad en } \\
\text { Internet y las cuestiones jurídicas }\end{array}$ & 3.46 & 1.02 & $3.58 \mathrm{a}$ & 0.91 & $3.35 \mathrm{a}$ & 1.12 & 3.52 & 0.95 & 3.42 & 1.08 \\
\hline $\begin{array}{l}8 \text { - Capacidad para construir y gestionar las redes } \\
\text { sociales virtuales }\end{array}$ & 3.91 & 1.12 & 3.95 & 1.07 & 3.87 & 1.18 & 3.93 & 1.09 & 3.9 & 1.15 \\
\hline
\end{tabular}

Los resultados de la encuesta se midieron en base a una escala de Likert de 0 a 5. En términos descriptivos, los participantes anotan baja en e-liderazgo $(\mathrm{M}=2.2) \mathrm{y}$ muestran el nivel de competencia moderada en el uso de herramientas electrónicas para apoyar la colaboración en equipo virtual $(\mathrm{M}=3,09)$, en la capacidad de adaptar su estilo de e-comunicativa $(M=3,5)$, en la capacidad de organizar un equipo virtual $(\mathrm{M}=3.47)$ y en la capacidad para gestionar la seguridad y asuntos legales de Internet $(\mathrm{M}=3,46)$. Los niveles más altos de competencia se encuentran en la capacidad de utilizar e-herramientas interactivas para comunicarse $(\mathrm{M}=4,01)$, la capacidad de busca y evaluar la información electrónica $(\mathrm{M}=3.91)$ y en la capacidad de construir y gestionar redes sociales virtuales $(M=3,97)$. En general, el nivel de competencia digital de los estudiantes "parece ser lo suficientemente bueno como para que puedan ser una parte productiva y activa del mundo laboral.

Las muestras se analizaron mediante pruebas t-test resultando sin diferencias significativas entre los participantes por área de estudio. Este es un resultado coherente con la definición de las competencias digitales como competencias básicas requeridas por todos los estudiantes URV.

Por sexos, este estudio demuestra que las mujeres tienen más probabilidades de obtener las competencias digitales que les permitan aprovechar las ventajas de las nuevas oportunidades del mercado laboral. Las mujeres son estadísticamente mejores que los hombres en su capacidad de utilizar las herramientas electrónicas para la comunicación on-line, su capacidad para adaptarse al estilo de la comunicación electrónica para adaptarse a diferentes públicos y en su capacidad para manejar los asuntos legales y de seguridad de Internet. 


\section{Conclusiones}

La competencia digital se presenta como un reto importante para las instituciones de educación superior. Consideramos por lo tanto, que las competencias digitales no solo abarcan la adquisición de las destrezas o habilidades en la utilización de las tecnologías; es decir, no sólo basta con saberlas usar sino más bien, saber el cuándo, el cómo y para qué utilizarlas. La capacidad de saber-hacer con las tecnologías digitales permitirá fortalecer las habilidades del estudiante y a su vez profundizar el conocimiento mediante la búsqueda de información, colaboración y la comunicación. En la mayoría de los casos, creemos que las universidades siguen pensando en el contenido y no se trata de reinventar las estrategias de enseñanza o al hablar de nuevas formas de desarrollo de las competencias y la evaluación.

Estos marcos de competencia y gran parte de lo que nuestros estudiantes aprenden hoy en día fueron diseñados para la era de papel. Necesitamos actualizar nuestros planes de estudio la competencia para la era digital. Se debe tomar en consideración el enfoque holístico del aprendizaje en medios electrónicos Arias-Oliva \& YáñezLuna (2013:29). Las universidades tienen que tomar la iniciativa de incluir las TIC y las herramientas Web 2.0 y 3.0 dentro del currículo educativo. De esta manera los estudiantes podrán adquirir las competencias digitales necesarias para trabajar en una sociedad completamente digital o bien, desarrollar sus habilidades tecnológicas en un entorno digital.

\section{Bibliografía}

AREA, M. \& GUARRO, A., (2012). "La alfabetización informacional y digital: fundamentos pedagógicos para la enseñanza y el aprendizaje competente". Revista española de Documentación Científica, (Monográfico), p.46-74.

ARIAS-OLIVA, M. \& YÁÑEZ-LUNA, J.C., (2013). "Elearning Evolution: New Concepts on ICT \& Learning". In T. WARD BYNUM et al., eds. ETHICOMP (2013). Conference Proceedings: The possibilities of ethical ICT. Kolding Campus, Denmark, p. 25-34.

BENSON, R. \& BRACK, C., (2009). "Developing the scholarship of teaching: what is the role of e-teaching and learning?". Teaching in Higher Education, 14(1), p.71-80.

BYUN, S. \& MILLS, J.E., (2011). "Exploring the creation of learner-centered e-training environments among retail workers: a model development perspective". Cyberpsychology, behavior and social networking, 14(1-2), p.65-69.

CABERO, J. ET AL., (2003). "Las nuevas tecnologías en la actividad universitaria". Pixel-Bit. Revista de Medios y Educación, 20, p.81-100.

CACCIAMANI, S. ET AL., (2012). "Influence of participation, facilitator styles, and metacognitive reflection on knowledge building in online university courses". Computers \& Education, 58, p.874-884. 
CAMACHO, M. \& LARA, T., (2011). M-Learning en España , Portugal y América Latina. Monográfico SCOPEO n.3, Salamanca: Servicios de publicaciones de la Universidad de Salamanca.

CARTELLI, A., (2010). "Frameworks for Digital Competence Assessment : Proposals, Instruments, and Evaluation". In Proceedings of Informing Science \& IT Education Conference (InSITE) (2010) Frameworks. p. 561-574.

CHISEGA-NEGRILA, A.-M., (2012). "Web 3.0 in Education". In The 8 th International Scientific Conference eLearning and software for Education. Bucharest , April 26-27, 2012, p. 455-460.

COETZEE, L. \& EKSTEEN, J., (2011). "The Internet of Things - Promise for the Future? An Introduction". IST-Africa 2011 Conference \& Exhibition, p.1-9.

DOMINGO, M. \& MARQUĖS, P., (2011). "Aulas 2.0 y uso de las TIC en la práctica docente". Comunicar. Revista Científica de Educomunicación, 19(37), p.169-175.

FERNÁNDEZ GÓMEZ, E., (2009). U-Learning: El futuro está aquí, Madrid: Alfaomega RA-MA.

GONZÁLEZ, C., (2010). "What do university teachers think eLearning is good for in their teaching?". Studies in Higher Education, 35(1), p.61-78.

GONZÁLEZ MARIÑO, J.C., (2008). "TIC y la transformación de la práctica educativa en el contexto de las sociedades del conocimiento". Revista de Universidad y Sociedad del Conocimiento, 5(2), p.1-8.

GUZMÁN FLORES, T. et al., (2011). "Formación docente para la integración de las TIC en la práctica educativa". Apertura: Revista de Innovación Educativa, 14(36), p.1-6.

HERMES LÜCK, E., (2009). "El proceso de transformación tecnológica y la formación docente". Revista de Universidad y Sociedad del Conocimiento, 6(1), p.1-10.

HUNT, I. et al., (2011). "Educational programmes for future employability of graduates in SMEs". Journal of Intelligent Manufacturing, (2004), p.1-10.

JETHRO, O.O., GRACE, A.M. \& THOMAS, A.K., (2012). "E-Learning and Its Effects on Teaching and Learning in a Global Age". International Journal of Academic Research in Business and Social Sciences, 2(1), p.203-210.

DE LA FUENTE ARIAS, J. ET AL., (2010). "Percepción del proceso de enseñanza-aprendizaje y rendimiento académico en diferentes contextos instruccionales de la Educación Superior". Psicothema, 22(4), p.806-812.

DE LA ORDEN HOZ, A., (2011). "El problema de las competencias en la educación general". Bordón, 63(1), p.47-61.

LEE, Y.-H., HSIEH, Y.-C. \& HSU, C.-N., (2011). "Adding Innovation Diffusion Theory to the Technology Acceptance Model: Supporting Employees' Intentions to use E-Learning Systems". Educational Technology \& Society, 14(4), p.124137.

LEHMANN, J. et al., (2013). "Finding News Curators in Twitter". In 22nd international conference on World Wide Web companion. p. 863-870. 
LÓPEZ DE LA MADRID, M.C. \& FLORES GUERRERO, K., (2006). "Análisis de competencias a partir del uso de las TIC". Apertura: Revista de Innovación Educativa, 6(5), p.36-55.

LOUREIRO, A., (2012). "Embracing Web 2.0 \& 3.0 tools to support lifelong learning - Let learners connect". Procedia - Social and Behavioral Sciences, 46, p.532-537.

MARQUES, A. \& BELO, O., (2011). "Discovering Student web Usage Profiles Using Markov Chains". The Electronic Journal of e-Learning, 9(1), p.63-74.

MARTÍNEZ ALVARADO, H., (2009). "La integración de las TIC en instituciones educativas". En R. CARNEIRO, J. C. TOSCANO, \& T. DÍAZ, (coord.). Los desafios de las TIC para el cambio educativo. Madrid: Santillana, p. 61-70.

MORRIS, R.D., (2011). "Web 3.0: Implications for Online Learning". TechTrends: Linking Research \& Practice to Improve Learning, 55(1), p.42-46.

O'REILlY, T., (2008). "Web 2.0 Compact Definition: Trying Again. O'Reilly Radar, p.1. Available at: http://radar.oreilly.com/2006/12/web-20-compact-definition-tryi.html [Accessed August 20, 2013].

PADMA, S. \& SESHASAAYEE, A., (2011). "Towards Maximum Spanning Tree Model in Web 3.0 Design and Development for Students using Discriminant Analysis". IJCSI International Journal of Computer Science Issues, 8(5), p.420-425.

PENALVA MARTÍNEZ, M.C., REY MÁS, C. \& LLINARES CISCAR, S., (2011). "Identidad y aprendizaje de estudiantes Análisis en un contexto b-Learning en didáctica de la matemática". Revista española de pedagogía, 69(248), p.101-118.

PRENDES ESPINOZA, M.P., (2007). "Internet Aplicado a la Educación: Estrategias, didácticas y Metodologías". En Nuevas Tecnologías Aplicadas a la Educación. Madrid: McGraw-Hill, p. 205-222.

REYES GONZÁLEZ, D.S.M. \& GUEVARA CRUZ, H., (2009). "Adopción de las tecnologías infocomunicacionales (TI) en docentes: actualizando enfoques". Revista Electrónica Teoría de la Educación. Educación y Cultura en la Sociedad de la Información, 10(1), p.134-150.

ROLDÁN, D. et al., (2010). Gestión de proyectos de E-Learning, Madrid: RA-MA, Alfaomega.

SANTIAGO CAMPIÓN, R. \& NAVARIDAS NALDA, F., (2012). "La Web 2.0 en escena". Pixel-Bit. Revista de Medios y Educación, 41, p.19-30.

STEVENS, K., (2008). “ Professional Education for e- Teaching in Networked Schools". In Proceedings of world academy of science, engineering and techno$\log y(2008)$. p. 425-430.

TORRES-CORONAS, T. et al., (2013). "Digital competences and entrepreneurial self-efficacy among graduate students". In ETHICOMP (2013). Conference Proceedings: The possibilities of ethical ICT. p. 470-477.

YEH, Y., (2010). "Analyzing Online Behaviors, Roles , and Learning Communities via Online Discussions". Educational Technology \& Society, 13(1), p.140-151. 


\section{Notas}

1 IoT acrónimo de Internet of Things (Internet de las cosas), que amplía el concepto "Any Time, Any Place for Any-One" en "Any Time, Any Place for Any-Thing

2 Nombre acuñado por Dale Dougherty y Craig Cline (O'Reilly y MediaLive International respectivamente), Tim O'Reilly desarrolló el concepto

3 Acrónimos de Leraning Management Systems Learning Content Management Systems, Entorno Virtual para la Enseñanza y el Aprendizaje, Grupos de Colaboración, Computer Supported Collaborative Learning y Computer Supported Collaborative Working, respectivamente.

4 Entiéndase por usuario como el destinatario final de las Tecnologías de Información y Comunicaciones, que puede ser un administrativo, docente o un estudiante en un contexto educativo.

\section{Los Autores}

Mario Arias Oliva es doctor en administración y dirección de empresas por la Universitat Rovira i Virgili y profesor titular en el departamento de gestión de empresas de la Universitat Rovira i Virgili. Ha publicado numerosos libros entre los que destacamos Encyclopedia of E-Human Resource Management (IGI, Estados Unidos) o Indicadores de la Sociedad de la Información (Instituto Nacional de Estadística, España). Ha presentado trabajos en más de treinta congresos internacionales y liderado numerosas investigaciones en el ámbito del e-management. Colabora como International Research Assosiate en el Center of Computy and Social Responsability (de Monfort University, UK). Ha obtenido entre otros premiso el de calidad docente del Ministerio de Educación y Ciencia (2002) y el premio a la excelencia docente en el área de Ciencias Sociales de la Universitat Rovira i Virgili en el año 2010.

Teresa Torres Coronas es Licenciada en Ciencias Económicas y Empresariales por la Universidad de Barcelona y doctora en Administración de Empresas por la Universidad Rovira i Virgili. Galardonada con el primer premio en la X edición del Premio EADA (2000). Autora de numerosos artículos y presentaciones en congresos sobre el campo de la gestión de intangibles, educación en la gestión, creatividad aplicada y TIC. Es una de los investigadores miembros del ELIS Group: E-government for Local Integration with Sustainability (Royal Holloway, University of London)

Juan Carlos Yáñez Luna es profesor titular de la materia Taller de computación en la Facultad de Economía de la Universidad Autónoma de San Luis Potosí. Se ha desempeñado en cargos administrativos como coordinador del Laboratorio de Cómputo dentro de la misma entidad. Ha colaborado en la realización de diversos cursos de mejoramiento al profesorado y a los estudiantes. Así como la instalación de una Espacio Virtual como apoyo a la actividad docente dela Facultad de Economía. Actualmente es estudiante de doctorado en la Universitat Rovira I Virgili (España) bajo la tutela del Prof. Arias-Oliva dentro de la línea de Investigación eLearning y mLearning y la aceptación de las tecnologías. 